\title{
FACTORIZATION AND ARITHMETIC FUNCTIONS FOR ORDERS IN COMPOSITION ALGEBRAS
}

\author{
by P. J. C. LAMONT
}

(Received 2 August, 1971)

A well-known product, referred to as the Dirichlet convolution product, is generalized to arithmetic functions defined on an order in a Cayley division algebra. Factorization results for orders, multiplicative functions and analogues of the Moebius inversion formula are discussed.

1. Introduction. Let $\mathbf{C}$, with nondegenerate quadratic form $N$, be a composition algebra over a field $k$ of characteristic other than 2. Then $N$ is a map of $\mathbf{C}$ into $k$ such that

(i) $N(t \xi)=t^{2} N(\xi)$ for all $t \in k$ and $\xi \in \mathbf{C}$,

(ii) $(\xi, \eta)=\frac{1}{2}\{N(\xi+\eta)-N(\xi)-N(\eta)\}$ is a bilinear function,

(iii) $N(\xi \eta)=N(\xi) N(\eta)$,

(iv) $(\xi, \eta)=0$ for all $\xi \in \mathbf{C}$ implies that $\eta=0$.

An algebra over $k$ satisfying the conditions

$$
\xi^{2} \eta=\xi(\xi \eta) \text { and } \xi \eta^{2}=(\xi \eta) \eta
$$

for all elements $\xi, \eta$ in the algebra is called alternative. It follows that $\xi(\eta \xi)=(\xi \eta) \xi$. $\mathbf{C}$ is simultaneously an alternative algebra with an involution $\xi \rightarrow \xi$ such that

$$
\xi \xi=N(\xi) 1 \text { and } \xi+\xi=2 T(\xi) 1, \text { where } N(\xi), T(\xi) \in k,
$$

and a quadratic algebra, since every element $\xi$ of $\mathbf{C}$ satisfies

$$
\xi^{2}-2 T(\xi) \xi+N(\xi) 1=0 .
$$

Since $\mathbf{C}$ is alternative, the Moufang identities hold:

$$
\begin{aligned}
(\xi \alpha)(\beta \xi) & =\xi(\alpha \beta) \xi, \\
(\xi \alpha \xi) \eta & =\xi[\alpha(\xi \eta)], \\
\eta(\xi \alpha \xi) & =[(\eta \xi) \alpha] \xi .
\end{aligned}
$$

I shall also use

$$
\begin{aligned}
T(\xi \eta) & =T(\eta \xi), \\
T[\xi(\eta \zeta)] & =T[(\xi \eta) \zeta] .
\end{aligned}
$$

Any alternative algebra with an identity and an involution that satisfies (1.2) is a composition algebra. 
Given a composition algebra $K$. Take $\mathbf{H}$ to be the direct sum $\mathbf{K} \oplus \mathbf{K} e$, where $\mathbf{K} e$ is isomorphic to $\mathbf{K}$ under $\xi \rightarrow \xi e$. Define multiplication in $\mathbf{H}$ by

$$
\left(\xi_{1}+\xi_{2} e\right)\left(\eta_{1}+\eta_{2} e\right)=\left(\xi_{1} \eta_{1}+a \bar{\eta}_{2} \xi_{2}\right)+\left(\eta_{2} \xi_{1}+\xi_{2} \bar{\eta}_{1}\right) e
$$

for $\mathbf{0} \neq a \in k$. Then $\mathbf{H}$ is a composition algebra if and only if $\mathbf{K}$ is associative. Also, any composition algebra over a field $k$ may be obtained from $k 1$ by applying this Dickson doubling process at most three times. Then $\mathbf{C}$ is one of the following: $k 1$; an algebra $k[e]$ with $e^{2}=a$; a generalized quaternion algebra; or a generalized Cayley algebra. $\mathbf{C}$ is a division algebra if and only if the norm $N$ is anisotropic.

Let the ground field be the rationals and take $i_{0}=1$. By letting $e=i_{1}, i_{2}$ and then $i_{4}$ with $a=-1$ each time and setting $i_{1} i_{2}=i_{3}, i_{1} i_{4}=i_{5}, i_{2} i_{4}=i_{6}$ and $i_{3} i_{4}=i_{7}$, we obtain a basis $\left\{i_{s}\right\}_{0}^{7}$ for the classical Cayley division algebra $\mathbf{D}$.

An order or arithmetic of a composition algebra $\mathbf{C}$ over a field with a ring of integers is, by definition, a not necessarily associative ring, consists of integral elements only and contains 1. Orders of $\mathbf{C}$ have been discussed for local and global fields by van der Blij and Springer [5]. To introduce composition algebras they allow a ground field of characteristic 2 .

If $\mathfrak{D}$ is an order of $\mathbf{D}$ and if $\mathrm{v}$ contains a subset of $\left\{\boldsymbol{i}_{s}\right\}$, then by closure of multiplication in $\mathfrak{o}$ the subset has cardinality $1,2,4$, or 8 . Examples of such orders, maximal in the algebra which they span, are: the rational integers $\mathbf{Z}$; the Gaussian integers $\mathbf{Z}[i]$; the Hurwitz quaternion order $Z\left[i_{1}, i_{2}, \rho\right]$ where $\rho=\frac{1}{2}\left(1+i_{1}+i_{2}+i_{3}\right)$; and the isomorphic maximal Cayley arithmetics [3]. The orders of $\mathbf{D}$ used below span the same algebra as does the subset of $\left\{i_{s}\right\}$ that they contain.

Let $\mathbf{v}$ be an order of $\mathbf{C}$ in which the number of representations of any integer by norms of elements of $\mathfrak{o}$ is finite. For $\xi$ and $\alpha \in \mathfrak{o}, \alpha$ is said to be a left divisor of $\xi$ if $\xi$ has a factorization $\alpha \beta$ in $\mathfrak{o}$, and we write $\alpha \mid \xi$. Right divisibility is similarly defined. Next one defines $r_{\mathrm{o}}(m)$ to be the number of elements of norm $m$ in $\mathfrak{v}$. For $\zeta \in \mathfrak{D}$ and $N \zeta=m n, s_{0}(\zeta, m, n)$ denotes the number of distinct factorizations $\delta \gamma$ of $\zeta$ in $\mathrm{o}$ with $N \delta=m$ and $N \gamma=n$. When no confusion can arise we omit the suffix $\mathrm{o}$ and write $r(1)=r$. Formulae for the values of the functions $r$ and $s$ on certain orders of $D$ are given in Rankin [4] and in [2]. The methods of proof used in [2] are reviewed in the next section.

2. Factorization. Let $\mathfrak{o}$ be an order of $\mathbf{D}$ which possesses the following properties:

(i) $r(m n) r=r(m) r(n)$ if $(m, n)=1$,

(ii) For any $\xi \in \mathfrak{o}$ of odd norm, there exists a unit $\varepsilon \in \mathfrak{o}$ such that $\xi \equiv \varepsilon(\bmod 2)$.

Then we are able to prove the theorem:

(2.3) Any element $\zeta \in \mathfrak{0}$ with $N \zeta=m n$ has precisely $r$ different factorizations $\xi_{\eta}$ in o with $N \xi=m$ and $N \eta=n$, if $(m, n)=1$. Moreover, for $m$ odd, the factorization is unique apart from signs if a unit $\varepsilon$ is prescribed to which $\xi$ is congruent modulo 2.

Proof. Suppose that $\zeta=\xi_{1} \eta_{1}=\xi_{2} \eta_{2}$, where $\xi_{1} \neq \pm \xi_{2}$ and $N \xi_{1}=N \xi_{2}=m$ is odd. 
Then $\left|T\left(\xi_{1} \xi_{2}\right)\right|<m$. By (1.1), $\zeta \bar{\eta}_{1}=n \xi_{1}$ and $\zeta \bar{\eta}_{2}=n \xi_{2}$. Hence $\left(\eta_{1} \bar{\zeta}\right)\left(\zeta \bar{\eta}_{2}\right)=n^{2} \xi_{1} \xi_{2}$. By (1.8), $m T\left(\eta_{1} \bar{\eta}_{2}\right)=n T\left(\xi_{1} \xi_{2}\right)$.

Assume that $\xi_{1} \equiv \xi_{2}(\bmod 2)$. Then $\xi_{1} \xi_{2} \equiv 1(\bmod 2)$. Hence $\xi_{1} \xi_{2}$ has integral coefficients with respect to $\left\{i_{s}\right\}_{0}^{7}$. Thus, since $m \mid T\left(\xi_{1} \xi_{2}\right)$, we have that $T\left(\xi_{1} \xi_{2}\right)=0$.

Again, since $N\left(\xi_{1} \xi_{2}\right)=m^{2}$, it follows that $\xi_{1} \xi_{2}$ has precisely one or five odd rational integral coefficients. Hence, using $(2.2), \xi_{1} \xi_{2} \equiv i_{s}(\bmod 2)$ for some $s(1 \leqq s \leqq 7)$. This is a contradiction. Therefore $\xi_{1} \not \equiv \xi_{2}(\bmod 2)$. Again by (2.2), $\zeta$ has at most $r$ factorizations $\xi \eta$.

Now consider all $\zeta \in \mathfrak{o}$ with norm $m n$. Suppose that there is some $\zeta \in \mathfrak{o}$ with strictly less than $r$ factorizations of the required form. Then

$$
r(m) r(n)<r \sum_{\zeta=m n} 1=r(m n) r .
$$

This contradicts (2.1). If $m$ is even, apply the argument to $s(\zeta, n, m)$. The theorem is thus proved.

Use of the alternative laws and the Moufang identities yields results of the following form.

(2.4). For $\varepsilon$ any unit in $\mathfrak{D}, s(\zeta, m, n)=s(\zeta \varepsilon, m, n)$.

Proof. If $\zeta=\xi \eta$, then $\zeta \varepsilon=\{[(\xi \bar{\varepsilon}) \varepsilon] \eta\} \varepsilon=(\xi \bar{\varepsilon})(\varepsilon \eta \varepsilon)$.

An element $\zeta \in \mathcal{O}$ of odd norm is called primitive if $\zeta \not \equiv 0(\bmod p)$ for any rational prime $p$. Suppose now that, for the order $\mathrm{D}$,

$$
r\left(p^{t+1}\right) r=r(p) r\left(p^{t}\right)+r\left(p^{t-1}\right)[r-r(p)]
$$

when the integer $t>0$. Then the following theorem holds:

(2.6). Any element $\zeta \in \mathcal{0}$, with $N \zeta=p^{t+1}$, where $p$ is an odd rational prime and the integer $t>0$, has precisely

(i) $r(p)$ distinct factorizations $\xi \eta$ with $N \xi=p$ and $N \eta=p^{t}$, if $\zeta \equiv 0(\bmod p)$,

(ii) $r$ such factorizations, if $\zeta$ is primitive.

Proof. (i) $\zeta=p \zeta^{\prime}$, where $\zeta^{\prime} \in \mathfrak{o}$. Let $\xi$ be any element of norm $p$ in $\mathfrak{o}$ and let $\eta=\xi \zeta^{\prime}$. Then $\xi \eta=\zeta$. Thus $\zeta$ has precisely as many distinct factorizations $\xi \eta$ of the required form as there are elements of norm $p$ in $\mathfrak{o}$.

(ii) Suppose that $\zeta$ has distinct factorizations $\xi_{1} \eta_{1}$ and $\xi_{2} \eta_{2}$ in o with $N \xi_{1}=N \xi_{2}=p$ and $\xi_{1} \neq \pm \xi_{2}$. Assume that $\xi_{1} \equiv \xi_{2}(\bmod 2)$. Then $\xi_{1} \xi_{2} \equiv 1(\bmod 2)$. Hence $\xi_{1} \xi_{2}$ has integral coefficients with respect to the basis $\left\{i_{s}\right\}_{0}^{7}$.

Now, by (1.7) and (1.8),

$$
T\left\{\xi_{1}\left(\xi_{2} \zeta\right)+\left(\zeta \xi_{1}\right) \xi_{2}\right\}=2 T(\zeta) T\left(\xi_{1} \xi_{2}\right) .
$$

Also $\xi_{1}\left(\xi_{2} \zeta\right)=p \xi_{1} \eta_{2}$ and $\left(\zeta \xi_{1}\right) \xi_{2}=p \bar{\eta}_{1} \xi_{2}$. Hence, using (2.4), $p$ divides $T\left(\xi_{1} \xi_{2}\right)$. Since $\xi_{1} \neq \pm \xi_{2}, \xi_{1} \xi_{2} \neq \pm p$. But $N\left(\xi_{1} \xi_{2}\right)=p^{2}$. Hence $T\left(\xi_{1} \xi_{2}\right)=0$ and $\xi_{1} \xi_{2}$ has precisely one or five odd rational integral coefficients. Thus $\xi_{1} \xi_{2}$ is congruent modulo 2 to one of $\left\{i_{s}\right\}_{1}$. This contradicts the fact that $\xi_{1} \xi_{2} \equiv 1(\bmod 2)$. Therefore $\xi_{1} \not \equiv \xi_{2}(\bmod 2)$. 
We have proved that there are at most $r$ distinct factorizations $\xi \eta$ of $\zeta$ in $\mathrm{o}$ with $N \xi=p$. Suppose that, for some $\zeta$ of norm $p^{t+1}$, there are less than $r$ such factorizations. Then

$$
\begin{aligned}
r(p) r\left(p^{t}\right) & <r \sum_{\substack{N \zeta=p^{t+1} \\
p \times \zeta}} 1+r(p) \sum_{\substack{N \zeta=p^{t+1} \\
p}} 1 \\
& =r\left(p^{t+1}\right) r-r\left(p^{t-1}\right) r+r(p) r\left(p^{t-1}\right) \\
& =r\left(p^{t+1}\right) r+r\left(p^{t-1}\right)[r(p)-r] .
\end{aligned}
$$

This contradicts (2.5) and completes the proof of the theorem.

An element $\xi \in \mathfrak{0}$ with $N \xi \neq 1$ is called irreducible if $\xi=\gamma \delta$ in $\mathfrak{v}$ implies that one of $\gamma$ and $\delta$ is a unit of $o$. If $\xi$ has norm a prime, then $\xi$ is irreducible.

Theorems (2.3) and (2.6) show that in, for example, the maximal Cayley arithmetics of D, unique factorization, apart from signs, order and parentheses, holds for primitive elements, provided that units are prescribed to which the irreducible factors are congruent modulo 2 and provided that parentheses are used in such a way that Theorem (2.6) is applicable.

Axiom (2.2) fails in the nonmaximal orders $\mathbf{J}_{1}=\mathbf{Z}\left[i_{1}, i_{2}, i_{3}\right]$ and $\mathbf{J}_{2}=\mathbf{Z}\left[i_{1}, \ldots, i_{7}\right]$. Factorization results for $J_{s}(s=1,2)$ may be deduced from (2.3) and (2.6). Consider congruence modulo 2 in corresponding maximal quaternion and Cayley orders. Note that, if $\xi \equiv \varepsilon(\bmod 2)$, then $\xi \in \mathbf{J}_{s}$ if and only if $\varepsilon \in \mathbf{J}_{s}$. Thus we need only consider factorizations corresponding to units of the orders $\mathbf{J}_{\mathbf{s}}$.

3. Arithmetic functions. Here the composition algebra $\mathbf{C}$, defined over the field of rational numbers, is assumed to be a division algebra. Again $\mathrm{o}$ is an order in $\mathbf{C}$ and $r_{0}(m)$ is finite for all integers $m$.

A function $f$ with domain $\mathfrak{o}$ and codomain the field of complex numbers is called arithmetic. Let $\mathfrak{A}$ denote the set of all arithmetic functions on $\mathfrak{o}$.

Suppose that $f$ and $g \in \mathfrak{A}$. A product $f \cdot g$ is defined by

$$
f \cdot g(\xi)=\frac{1}{r} \sum_{\delta \mid \xi} f(\delta) g\left(\delta^{-1} \xi\right),
$$

where the sum extends over all left divisors $\delta$ of $\xi$ in $\mathfrak{v}$. Then $f \cdot g \in \mathfrak{Y}$. Also

$$
f \cdot g(\xi)=\frac{1}{r} \sum_{\alpha \beta=\xi} f(\alpha) g(\beta),
$$

where the summation is over all ordered pairs $\alpha, \beta$ of elements of $\mathrm{o}$ with the product $\alpha \beta$ equal to $\xi$.

First we consider the following symmetry properties:

$$
\begin{gathered}
f(\varepsilon \xi)=f(\xi \varepsilon)=f(\xi) \text { for all } \xi \text { and units } \varepsilon \in \mathfrak{D}, \\
f(\xi)=f(\xi) \text { for all } \xi \in \mathfrak{D} .
\end{gathered}
$$

Let $\mathfrak{U}_{1}$ be the set of all elements in $\mathfrak{O}$ satisfying (3.3). 
(3.5). $\mathfrak{U}_{1}$ is closed.

Proof. Take $f, g \in \mathfrak{A}_{1}$ and $\xi, \varepsilon \in \mathrm{o}$ with $\varepsilon$ a unit. Using the Moufang identity (1.5), we have

$$
f \cdot g(\varepsilon \xi)=\frac{1}{r} \sum_{\alpha \beta=\varepsilon \xi} f(\alpha) g(\beta)=\frac{1}{r} \sum_{\tilde{\varepsilon}\{\alpha[\varepsilon(\varepsilon \beta)]\}=\xi} f(\alpha) g(\beta)=\frac{1}{r} \sum_{(\bar{\varepsilon} \alpha \bar{\varepsilon})(\varepsilon \beta)=\xi} f(\bar{\varepsilon} \alpha \bar{\varepsilon}) g(\varepsilon \beta)=f \cdot g(\xi) .
$$

Now from (1.4) we deduce that

$$
f \cdot g(\varepsilon \xi)=\frac{1}{r} \sum_{(\bar{\varepsilon} \alpha)(\beta \bar{\varepsilon})=\xi \bar{\varepsilon}} f(\bar{\varepsilon} \alpha) g(\beta \bar{\varepsilon})=f \cdot g(\xi \bar{\varepsilon}) .
$$

Thus $f \cdot g \in \mathfrak{Q}_{1}$.

Now suppose that functions $f$ and $g \in \mathfrak{I}$ satisfy (3.4). Then

$$
f \cdot g(\bar{\zeta})=\frac{1}{r} \sum_{\gamma \delta=\xi} f(\gamma) g(\delta)=\frac{1}{r} \sum_{\alpha \beta=\xi} g(\alpha) f(\beta)=g \cdot f(\xi) .
$$

For an arithmetic function $f, f^{*}$ is defined to be the restriction of $f$ to the integers $\mathbf{Z}$. For $f, g$ and $h \in \mathfrak{U}$ we have $f \cdot g^{*}=g \cdot f^{*}$ and $(f \cdot g) \cdot h^{*}=f \cdot(g \cdot h)^{*}$.

Now define a function $e$ by

$$
e(\xi)=\left\{\begin{array}{ll}
1 & \text { if } \xi \text { is a unit } \\
0 & \text { otherwise. }
\end{array}\right\}
$$

Clearly $e \in \mathfrak{U}_{1}$ and $e$ satisfies (3.4). Now for $f \in \mathfrak{I}_{1}$ we have

$$
f \cdot e(\xi)=\frac{1}{r} \sum_{\alpha \beta=\xi} f(\alpha) e(\beta)=\frac{1}{r} \sum_{N \varepsilon=1} f(\xi \bar{\varepsilon})=f(\check{\zeta}) .
$$

Similarly $e \cdot f=f$. Hence $e$ is the unique identity for $\mathfrak{A}_{1}$.

(3.7). For $f \in \mathfrak{A}_{1}$, a right inverse $f^{\prime} \in \mathfrak{P}_{1}$ exists if and only if $f(1) \neq 0$.

Proof. Note that, if $f \in \mathfrak{U}_{1}$, then $f(\varepsilon)=f(1)$ for all units $\varepsilon \in \mathbf{0}$. Suppose that $f^{\prime} \in \mathfrak{Y}_{1}$ exists. Then $1=e(1)=f \cdot f^{\prime}(1)=f(1) f^{\prime}(1)$, by (3.2) and (3.3). Therefore $f(1) \neq 0$.

Now assume that $f(1) \neq 0$. Define $f^{\prime}$ inductively as follows.

$$
f^{\prime}(\xi)=\left\{\begin{array}{l}
{[f(1)]^{-1}, \text { if } \xi \text { is a unit, }} \\
-[r f(1)]^{-1} \sum_{\substack{\alpha \beta=\xi \\
\alpha \alpha \neq 1}} f(\alpha) f^{\prime}(\beta), \text { otherwise. }
\end{array}\right\}
$$

An induction argument using the Moufang identities (1.5) and (1.6) shows that $f^{\prime} \in \mathfrak{R}_{1}$. Next, for $\varepsilon$ a unit,

$$
f \cdot f^{\prime}(\varepsilon)=\frac{1}{r} \sum_{N \varepsilon_{1}=1} f\left(\varepsilon_{1}\right) f^{\prime}\left(\bar{\varepsilon}_{1} \varepsilon\right)=\frac{1}{r} \sum_{N \varepsilon_{1}=1} f(1) f^{\prime}(1)=1 .
$$


For $\xi$ with $N \xi \neq 1$, we have

$$
\begin{aligned}
& f \cdot f^{\prime}(\xi)=\frac{1}{r} \sum_{\alpha \beta=\xi} f(\alpha) f^{\prime}(\beta)
\end{aligned}
$$

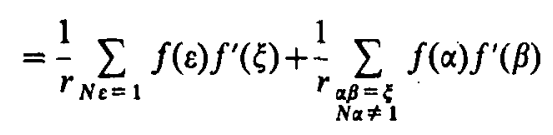

$$
\begin{aligned}
& =f(1) f^{\prime}(\xi)-f(1) f^{\prime}(\xi)=0 .
\end{aligned}
$$

Hence $f \cdot f^{\prime}=e$ and $f^{\prime}$ is a right inverse for $f$.

If $f \in \mathscr{U}_{1}$ satisfies (3.4), then $f^{\prime}$ is a left inverse for $f$ under the convolution product defined by

$$
f \times g(\xi)=\frac{1}{r} \sum_{\alpha \beta=\xi} f(\beta) g(\alpha)
$$

with summation as before. If $f \in \mathscr{R}_{1}$ and both $f$ and $f^{\prime}$ satisfy (3.4), then

$$
f^{\prime} \cdot f(\xi)=f \cdot f^{\prime}(\xi)=e(\xi)=e(\xi) .
$$

Hence $f^{\prime} \cdot f=e$ and $f^{\prime}$ is also a left inverse.

4. Multiplicative functions. Let $\mathrm{o}$ again be an order of the Cayley division algebra over the rationals. We consider orders $\mathrm{o}$ in which, for $N \zeta=u v, s(\zeta, u, v)=r$ if $(u, v)=1$ or if $\zeta$ is primitive.

An arithmetic function $f$ on $v$ is said to be multiplicative if it possesses the property

$$
f(\xi \eta)=f(\xi) f(\eta)
$$

when $\xi, \eta \in \mathfrak{O}$ and $(N \xi, N \eta)=1$. Let $\mathfrak{M}$ denote the set of all nonzero multiplicative functions in $\mathfrak{I}_{1}$.

(4.2). If $f$ and $g \in \mathfrak{M}$, then $f \cdot g^{*} \in \mathfrak{N}$.

Proof.

$$
f \cdot g(1)=\frac{1}{r} \sum_{N \varepsilon=1} f(\varepsilon) g(\bar{\varepsilon})=1
$$

Now take positive integers $m$ and $n$ with $(m, n)=1$.

$$
f \cdot g(m n)=\frac{1}{r} \sum_{\xi \mid m n} f(\xi) g\left(\xi^{-1} m n\right)
$$

We may write $\xi=\xi_{1} \xi_{2}$ in any one of $r$ ways where $\xi_{1} \mid m$ and $\xi_{2} \mid n$ and $N \xi_{1}$ and $N \xi_{2}$ are fixed. Conversely, by (1.7) and (1.8), if $\xi_{1} \mid m$ and $\xi_{2} \mid n$, then $\xi_{1} \xi_{2} \mid m n$. Hence

$$
f \cdot g(m n)=\frac{1}{r^{2}} \sum_{\substack{\xi_{1} \\ \xi_{2}}} f\left(\xi_{1} \xi_{2}\right) g\left(\xi_{2}^{-1} n \xi_{1}^{-1} m\right)=f \cdot g(m) f \cdot g(n) .
$$


For $\xi, \eta \in \mathfrak{0}, \xi \sim \eta$ means that $N \xi=N \eta$ and, for $p \in Z, p^{m} \mid \xi$ if and only if $p^{m} \mid \eta$. Take $\xi \sim \eta$ in $\mathfrak{v}$. Let $\beta, \gamma$ be any left (or right) divisors of $\xi, \eta$, respectively, in 0 , such that $\beta \sim \gamma$. If $s(\beta, u, v)=s(\gamma, u, v)$ for all positive integers $u, v$ with $u v$ equal to the common norm, we shall write $s(\xi)=s(\eta)$. Henceforth it is assumed that, in $0, \xi \sim \eta$ implies that $s(\xi)=s(\eta)$.

I shall consider functions $f$ satisfying the condition

$$
f\left(\xi_{1}\right)=f\left(\xi_{2}\right) \text { if } \xi_{1} \sim \xi_{2} .
$$

(4.4). If arithmetic functions $f$ and $g$ satisfy (4.3), then so does $f \cdot g$. Then

Proof. Take $\xi_{1} \sim \xi_{2}$. Let there be $t$ integral elements $\beta$ of fixed norm $n$ such that $\beta \mid \xi_{1}$.

$$
s\left(\xi_{1}, n, n^{-1} N \xi_{1}\right)=s\left(\xi_{2}, n, n^{-1} N \xi_{2}\right)
$$

Therefore there are $t$ elements $\gamma \in \mathcal{O}$ of norm $n$ that are left divisors of $\xi_{2}$. Also, if $p^{m} \mid \beta$, then $p^{m} \mid \xi_{1}$, and hence $p^{m} \mid \xi_{2}$. Let $\xi_{s}=p^{m} \xi_{s}^{\prime}(s=1,2)$. Then

$$
s\left(\xi_{1}^{\prime}, n p^{-2 m}, n^{-1} N \xi_{1}\right)=s\left(\xi_{2}^{\prime}, n p^{-2 m}, n^{-1} N \xi_{2}\right) \text {. }
$$

Hence there is a one-to-one correspondence between the elements $\beta$ and the elements $\gamma$ under which $\beta \sim \gamma$. A similar result holds for the corresponding right divisors.

$$
f \cdot g\left(\xi_{1}\right)=\frac{1}{r_{\beta} \mid \xi_{1}} \sum_{1} f(\beta) g\left(\beta^{-1} \xi_{1}\right)=\frac{1}{r} \sum_{\gamma \mid \xi_{2}} f(\gamma) g\left(\gamma^{-1} \xi_{2}\right)=f \cdot g\left(\xi_{2}\right) .
$$

(4.5). If an arithmetic function $h$ satisfies (4.3) and if $h(1) \neq 0$, then $h^{\prime} \in \mathfrak{U}_{1}$ exists and satisfies (4.3).

Proof. For $\varepsilon$ a unit of $\mathfrak{o}, h^{\prime}(\varepsilon)=[1 / h(1)]=h^{\prime}(1)$. Assume that (4.3) holds for $h^{\prime}$ whenever $N \xi_{1}=N \xi_{2}<N \xi$. Take $\xi \sim \eta$. Then, as in the proof of (4.4),

$$
h^{\prime}(\xi)=\frac{-1}{r h(1)} \sum_{\substack{\alpha \beta=\xi \\ N \alpha \neq 1}} h(\alpha) h^{\prime}(\beta)=\frac{-1}{r h(1)} \sum_{\substack{\delta \delta=\eta \\ N \gamma \neq 1}} h(\gamma) h^{\prime}(\delta)=h^{\prime}(\eta) .
$$

Let $\mathfrak{D}_{1}$ be the set of all functions $f \in \mathfrak{D}$ satisfying (4.3).

(4.6). If $f$ and $g \in \mathfrak{M}_{1}$, then

$$
f \cdot g(\xi \eta)=f \cdot g(\xi) f \cdot g(\eta)
$$

if $\xi$ and $\eta$ are primitive in $\mathrm{o}$ and if $(N \xi, N \eta)=1$.

$f \cdot g$ is then said to be multiplicative on primitive elements.

$$
\text { Proof: } f \cdot g\left(\xi_{\eta}\right)=\frac{1}{r} \sum_{\delta \mid \xi \eta} f(\delta) g\left[\delta^{-1}(\xi \eta)\right] \text {. }
$$

Suppose that $\xi, \eta \in \mathcal{0}$ are primitive and that $(N \xi, N \eta)=1$. For fixed norm $n$ there are $r$ left divisors $\delta$ of $\xi \eta$ with $N \delta=n$ provided that $n \mid N(\xi \eta)$. Let $\delta=\alpha \beta$, where $N \alpha \mid N \xi$ and 
$N \beta \mid N \eta$. There are $r$ such factorizations of each $\delta$ for fixed $N \alpha$ and $N \beta$. Now $\xi=\alpha_{1} \xi_{1}$ and $\eta=\beta_{1} \eta_{1}$ each in any one of $r$ ways where $N \alpha_{1}=N \alpha, N \beta_{1}=N \beta$. Then $\alpha_{1} \sim \alpha$ and $\beta_{1} \sim \beta$. Also $(\alpha \beta)^{-1}(\xi \eta) \sim\left(\alpha_{1}^{-1} \xi\right)\left(\beta_{1}^{-1} \eta\right)$.

$$
f \cdot g(\xi \eta)=\frac{1}{r^{2}} \sum_{\alpha \beta \mid \xi \eta \eta} f(\alpha \beta) g\left[(\alpha \beta)^{-1}(\xi \eta)\right]=\frac{1}{r^{2}} \sum_{\substack{\alpha_{1}\left|\xi \\ \beta_{1}\right| \eta \eta}} f\left(\alpha_{1} \beta_{1}\right) g\left[\left(\alpha_{1}^{-1} \xi\right)\left(\beta_{1}^{-1} \eta\right)\right]=f \cdot g(\xi) f \cdot g(\eta) .
$$

For any complex number $x$, arithmetic functions $n_{x}$ are defined by $n_{x}(\xi)=(N \xi)^{x}$. Then $n_{x} \in \mathfrak{M}_{1} \subseteq \mathfrak{M}$.

(4.7) Let $g(\xi)=\frac{1}{r} \sum_{\delta \mid \xi} f(\delta)$.

(i) If $f \in \mathfrak{M}$, then $g^{*} \in \mathfrak{M}$.

(ii) If $f \in \mathfrak{M}_{1}$, then $g$ is multiplicative on primitive elements.

Proof. $g=f \cdot n_{0}$. The results follow by (4.2) and (4.6) respectively.

(4.8). If $f \in \mathfrak{M}_{1}$, then $f^{\prime}$ is multiplicative on primitive elements.

Proof. $f^{\prime}$ exists and satisfies (4.3). $f^{\prime}(\varepsilon)=1$ for any unit $\varepsilon \in \mathcal{O}$.

Assume that $f^{\prime}(\alpha \beta)=f^{\prime}(\alpha) f^{\prime}(\beta)$ for all primitive $\alpha, \beta \in \mathcal{D}$ with $(N \alpha, N \beta)=1$ and $N(\alpha \beta)<b$. Choose primitive $\xi, \eta \in 0$ with $(N \xi, N \eta)=1$ and $N \xi N \eta=b$. Then

$$
\begin{aligned}
0 & =e(\xi \eta)=f \cdot f^{\prime}(\xi \eta) \\
& =\frac{1}{r} \sum_{\delta \mid \xi \eta} f(\delta) f^{\prime}\left[\delta^{-1}(\xi \eta)\right] \\
& =\frac{1}{r^{2}} \sum_{\substack{\alpha \\
\beta}} f(\alpha \beta) f^{\prime}\left[\left(\alpha^{-1} \xi\right)\left(\beta^{-1} \eta\right)\right]+\frac{1}{r^{2}} \sum_{N e_{1} \varepsilon_{2}=1} f^{\prime}(\xi \eta) \\
& =\frac{1}{r^{2}} \sum_{\alpha \mid \xi} f(\alpha) f^{\prime}\left(\alpha^{-1} \xi\right) \sum_{\beta \mid \eta} f(\beta) f^{\prime}\left(\beta^{-1} \eta\right)-f^{\prime}(\xi) f^{\prime}(\eta)+f^{\prime}(\xi \eta) \\
& =f \cdot f^{\prime}(\xi) f \cdot f^{\prime}(\eta)-f^{\prime}(\xi) f^{\prime}(\eta)+f^{\prime}(\xi \eta) .
\end{aligned}
$$

Thus $f^{\prime}(\xi \eta)=f^{\prime}(\xi) f^{\prime}(\eta)$.

5. Moebius inversion. Let $\mu=n_{0}^{\prime}$. Then $\mu$ is multiplicative on primitive elements and on $\mathbf{Z}$.

(5.1). If $\xi \in \mathcal{0}$ and $N \xi>1$, then $\sum_{\eta \uparrow \xi} \mu\left(\eta^{-1} \xi\right)=0$.

Proof. $\frac{1}{r_{\eta \mid \xi}} \sum_{\xi} \mu\left(\eta^{-1} \xi\right)=\frac{1}{r_{\eta \mid \xi}} \sum_{0} n_{0}(\eta) \mu\left(\eta^{-1} \xi\right)=n_{0} \cdot \mu(\xi)=e(\xi)=0$. 
(5.2). $\mu(\xi)=-1$, if $\xi$ is irreducible in $\mathrm{o}$.

Proof. $0=\frac{1}{r} \sum_{\delta \mid \xi} \mu\left(\delta^{-1} \xi\right)=\frac{1}{r} \sum_{N=1} \mu(\xi)+\frac{1}{r_{N}} \sum_{\varepsilon=1} \mu(\varepsilon)=\mu(\xi)+1$.

Hence

(5.3). If $\eta$ is any product of $t$ primitive irreducible elements of 0 with distinct norms, then $\mu(\eta)=(-1)^{t}$.

Now it is easy to prove

(5.4). For rational prime $p, \mu(p)=(1 / r) r(p)-1$.

Proof. $0=\frac{1}{r} \sum_{\delta \mid p} \mu(\delta)=\frac{1}{r} \sum_{N \varepsilon=1} \mu(\varepsilon)+\frac{1}{r} \sum_{N \delta=p} \mu(\delta)+\frac{1}{r} \sum_{N=1} \mu(p)=1-\frac{1}{r} r(p)+\mu(p)$.

We recall that, in $\mathbf{Z}, r(p)=0$ and, in the Gaussian integers, for odd $p$,

$$
r(p)=4\left\{1+(-1)^{\frac{1}{2}(p-1)}\right\} .
$$

(5.5). If $N \zeta=p^{2}$, then $\mu(\zeta)=(1 / r) s(\zeta, p, p)-1$.

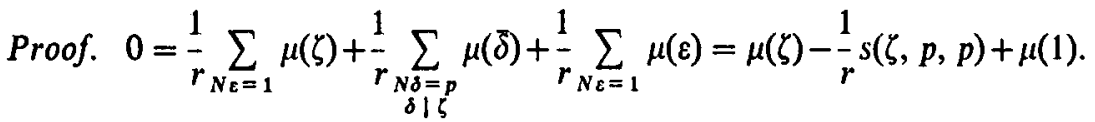

(5.6). If $N \zeta=p^{k}$, where $\zeta$ is primitive, $p$ is a prime and $k \geqq 2$, then $\mu(\zeta)=0$.

Proof. For $k=2, \mu(\zeta)=0$ by (5.5). The result follows by induction.

$\mu$ defined on $\mathbf{Z}$ is the well-known Moebius function. For $\mu$ defined on $\mathfrak{o}$, the following inversion formula holds.

(5.7). Under any condition or restriction that makes the convolution product associative and for any arithmetic functions $f$ and $g \in \mathfrak{Q}_{1}$,

$$
g(\xi)=\frac{1}{r} \sum_{\eta \mid \xi} f(\eta) \text { if and only if } f(\xi)=\frac{1}{r} \sum_{\eta \mid \xi} g(\eta) \mu\left(\eta^{-1} \xi\right) .
$$

Proof. $g=f \cdot n_{0}$. Thus $g \cdot \mu=f$. Conversely, by (4.5), $\mu$ satisfies (3.4) and is therefore a left inverse for $n_{0}$.

The theorem may be generalized by replacing $n_{0}$ by any function $h \in \mathfrak{A}_{1}$ with an inverse $h^{\prime}$, provided that the function and the inverse satisfy (3.4). Any $h \in \mathfrak{Q}_{1}$ that satisfies (4.3) and has $h(1) \neq 0$ would be suitable.

\section{REFERENCES}

1. N. Jacobson, Composition algebras and their automorphisms, Rend. Circ. Mat. Palermo 7 (1958), 55-80. (1962).

2. P. J. C. Lamont, On arithmetics in Cayley's algebra and multiplicative functions, Thesis, Glasgow 
3. P. J. C. Lamont, Arithmetics in Cayley's algebra, Proc. Glasgow Math. Assoc. 6 (1963), 99-106.

4. R. A. Rankin, A certain class of multiplicative functions, Duke Math. J. 13 (1946), 281-306.

5. F. van de Blij and T. A. Springer, The arithmetics of the octaves and of the group $G_{2}, N e d e r l$. Akad. Wetensch. Proc. 62 A (1959), 406-418.

St. Mary's College

and

UNIVERSITY OF NOTRE. DAME

Notre Dame, Indiana 46556 